\title{
Readmission After Pancreatectomy for Pancreatic Cancer in Medicare Patients
}

\author{
Deepthi M. Reddy • Courtney M. Townsend Jr. • \\ Yong-Fang Kuo • Jean L. Freeman • \\ James S. Goodwin • Taylor S. Riall
}

Received: 24 May 2009/Accepted: 25 August 2009 / Published online: 16 September 2009

(C) 2009 The Author(s). This article is published with open access at Springerlink.com

\begin{abstract}
Objective The objective of this study was to use a population-based dataset to evaluate the number of readmissions and reasons for readmission in Medicare patients undergoing pancreatectomy for pancreatic cancer.

Methods We used Surveillance, Epidemiology, and End Results-Medicare linked data (1992-2003) to evaluate the initial hospitalization, readmission rates within 30 days (early), and between 30 days and 1 year (late) after initial discharge and reasons for readmission in patients 66 years and older undergoing pancreatectomy.

Results We identified 1,730 subjects who underwent pancreatectomy for pancreatic cancer. The in-hospital mortality was $7.5 \%$. The overall Kaplan-Meier readmission rate was $16 \%$ at 30 days and 53\% at 1 year, accounting for 15,409 additional hospital days. Early readmissions were clearly related to operative complications in $80 \%$ of cases and unrelated diagnoses in $20 \%$ of cases. Late readmissions were related to recurrence in $48 \%$, operative complications in $25 \%$, and unrelated diagnoses in $27 \%$ of cases. In a multivariate analysis, only distal pancreatic resection $(P=0.02)$ and initial postoperative length of stay $\geq 10$ days $(P=0.03)$ predicted early readmission. When compared to patients not readmitted, patients readmitted early had worse median survival (11.8 vs.16.5 months, $P=0.04)$, but the 5 -year survival was identical $(18 \%)$. Late readmission was associated with worse median and 5-year survival $(19.4$ vs. 12.1 months, $12 \%$ vs. $21 \%, P<0.0001)$. Conclusions Our study demonstrates overall 30 -day and 1-year readmission rates of $16 \%$ and $53 \%$. The majority of early readmissions were related to postoperative complications but not related to patient and tumor characteristics. Complications causing early readmission are a cause of early mortality and are potentially preventable. Conversely, late readmissions are related to disease progression and are a marker of early mortality and not the cause.
\end{abstract}

Keywords Readmission · Pancreatic resection .

Kaplan-Meier. Operative complications

D. M. Reddy $\cdot$ C. M. Townsend Jr. T. S. Riall $(\square)$

Department of Surgery, University of Texas Medical Branch,

301 University Boulevard,

Galveston, TX 77555-0542, USA

e-mail: tsriall@utmb.edu

Y.-F. Kuo • J. L. Freeman • J. S. Goodwin

Department of Internal Medicine,

The University of Texas Medical Branch,

Galveston, TX, USA

\section{Introduction}

Over the last three decades, the operative mortality and lengths of stay have decreased following pancreatic resection, ${ }^{1-6}$ which can be attributed to increasing regionalization of care, ${ }^{7-10}$ improved perioperative and critical care, ${ }^{11-13}$ improved prevention and management of complications, implementation of critical pathways, ${ }^{14,15}$ and improved post-hospital inpatient and outpatient care. Despite the improvements in mortality and lengths of stay, the morbidity rates, usually defined as the occurrence of any complication in the postoperative period, remain high with reported rates in excess of $30 \%$ even at major centers. ${ }^{3,4,6,16-20}$ Readmission, a good measure of morbidity, is rarely reported. In addition, when reported, the focus is on readmissions within the first year. 
There are three previous studies evaluating readmission following pancreatic surgery. ${ }^{16,21,22}$ Two studies are singleinstitution studies, both of which included pancreaticoduodenectomy for benign and malignant disease. ${ }^{16,21}$ Neither study reported readmissions within 30 days of discharge. Emick and colleagues ${ }^{16}$ reported a $19 \%$ readmission rate in the year after surgery in 1,643 patients undergoing pancreaticoduodenectomy. van Geenen and colleagues ${ }^{21}$ reported an overall 1 year readmission rate of $38 \%$ in 283 patients undergoing pancreaticoduodenectomy. Given the single-institution nature of these studies, readmissions to other facilities may not be identified, so the reported rates may not reflect national readmission rates.

A population-based study using the California tumor registry and hospital discharge data reports a $59 \%$ readmission rate in the year after pancreaticoduodenectomy in patients with pancreatic cancer. ${ }^{22}$ They also report decreased long-term survival in the group requiring readmission. The majority of readmissions were related to disease progression. As such, they are a marker of early mortality and not the cause. None of the above studies evaluated readmissions using a time-to-event analysis and therefore potentially underestimated readmission rates.

The goals of our study were to use a population-based data set [Surveillance, Epidemiology, and End Results (SEER)-Medicare-linked data] to evaluate the readmission rates using time-to-event methods. We also evaluate the reasons for readmission within 30 days of pancreatic resection (early readmission) and between 30 days and 1 year (late readmissions). We hypothesize that early readmissions are related to operative complications, contribute to early mortality, and are potentially preventable. Conversely, late readmissions are associated with disease progression and are a marker, rather than a cause, of early mortality. Therefore, it is critical to analyze 30-day readmissions separately. We also determine the patient and tumor factors associated with early readmission and perform a survival analysis to determine the effect of early and late readmission on survival.

\section{Methods}

This study was approved by the Institutional Review Board at the University of Texas Medical Branch at Galveston. A Data Use Agreement for the use of SEER-Medicare data has been signed.

\section{Data Source}

We used data from the SEER-Medicare Linked Data Project (SMLDP) for the analysis. The SEER tumor registry is a National Cancer Institute (NCI) program, which tracks the incidence of cancer in the USA. The SEER database contains information on patient demographics, tumor characteristics, first course of treatment, and survival data (obtained via linkage to the National Death Index). From 1992-1999, SEER was comprised of 14 registries, 12 of which participated in the SEER-Medicare linkage. After 2000, SEER had 18 registries, 16 of which participated in the SEER-Medicare linkage. ${ }^{23,24}$

The SMLDP includes the SEER program, the NCI, and the Centers for Medicare and Medicaid Services). Ninetythree percent of all SEER patients older than age 65 are matched with Medicare enrollment files. In addition to the variables available in SEER, claims data for hospital stays, physician services, and hospital outpatient visits are included. The data used in this proposal include SEER subjects through 2002 and their Medicare claims through 2003.

\section{Patient Cohort Selection}

Using the SEER-Medicare-linked data, the following subjects were included in the study: (1) patients with International Classification of Diseases (ICD)-O-3 histology codes consistent with adenocarcinoma to eliminate other pancreatic tumor types such as neuroendocrine and acinar cell cancers, (2) patients diagnosed between 1992 and 2002, (3) patients with a pancreatic cancer as their first primary cancer, (4) patients enrolled in both Medicare Parts $\mathrm{A}$ and $\mathrm{B}$ without HMO for 12 months before their cancer diagnosis and for 1 year after their diagnosis, (5) patients aged $\geq 66$ (to ensure available Medicare claims data for a full year prior to diagnosis), and (6) patients undergoing pancreatic resection (complete resection of the primary tumor). Pancreatic resection was identified by searching MEDPAR inpatient claims files for ICD-9 CM codes for total pancreatectomy, radical pancreaticoduodenectomy, proximal pancreatectomy, distal pancreatectomy, radical subtotal pancreatectomy, or other partial pancreatectomy (codes shown in Table 1). Patients diagnosed at autopsy only or patients diagnosed by death certificate only were excluded.

\section{Assessment of Readmissions and Diagnoses}

We defined readmission as the number of patients who were discharged from an acute care hospital and readmitted to the hospital within (1) 30 days (early) or (2) between 30 days and 1 year (late) from the date of discharge from the index admission for pancreatic resection. To account for a decrease in the number of patients at risk in each time period as a result of tumor- and operative-related deaths, a Kaplan-Meier analysis modeling the time to readmission was used to obtain accurate readmission rates. We cannot directly identify patients that were transferred from one 
Table 1 ICD-9 Procedure and Diagnosis Codes

Procedure
Total pancreatectomy
Radical pancreaticoduodenectomy
Proximal pancreatectomy
Distal pancreatectomy
Radical subtotal pancreatectomy
Other partial pancreatectomy
Upper endoscopy with or without intervention
Biliary drainage via percutaneous,
endoscopic, or operative approach
Diagnosis
Operative complications

Metastatic disease

Dehydration

Gastric outlet obstruction/delayed gastric emptying Venous thromboembolism/ pulmonary embolism Pneumonia

Cholangitis

Small bowel obstruction

Gastritis/duodenitis/gastric ulcer/ duodenal ulcer

Incisional hernia

Pancreatic pseudocyst

Acute myocardial infarction

Cerebrovascular accident

Bile leak

Hip fracture
ICD-9 procedure codes

52.7

52.6

52.51

52.52

52.53

52.29

$44.13,44.12,44.14,44.19,44.12,44.22,44.43,45.13,45.16$, $45.22,45.23,45.24,45.25,45.28,45.29,45.30,54.59$

$51.98,51.87,51.10,51.11,51.86,51.85,57.84,51.1,51.31,51.51$, $51.59,51.3,51.32,51.42,51.37,51.34,51.43,51.49,87.51$

ICD-9 Diagnosis Codes

998, 998.0, 998.11, 998.12, 998.13, 998.3, 998.30, 998.31, 998.32, 998.4, 998.51, 998.59, 998.6, 998.83, 998.89,

$998.9,997.4,997.5,997.9$

197, 197.0, 197.1, 197.2, 197.3, 197.4, 197.5, 197.6, 197.7, $197.8,197.9,198,198.0,198.1,198.2,198.3,198.4,198.5$, 198.6, 198.7, 198.8, 198.81, 198.82, 198.89, 196.1, 196.2, 196.3, 196.4, 196.5, 196.6, 196.7, 196.8, 196.9, 199, 199.0, $199.1,789.5$

$276,276.0,276.2,276.4,276.5,276.50,276.51,276.52$

$537,537.0,537.3,537.89,537.9,536.3,536.8,536.9$

$453.8,444.21,444.42,453.1,453.2,453.40,453.4,453.42,453.9,415.19$

480.0, 480.1, 480.2, 480.3, 480.8, 480.9, 481, 481.0, 482.0, 482.1, 482.2, 482.30, 482.31, 482.32, 482.39. 482.40, 482.41, 482.42, $482.49,482.81,482.82,482.83,482.84,482.89,482.9,483.0,483.1$, 483.8, 484.1, 484.3, 484.5, 484.6, 484.7, 484.8, 487.0, 486, 485

$576.1,576.2,576.8,576.9,572$

$560.2,560.81,560.89,560.9$

$531.00,531.01,531.10,531.11,531.20,531.21,531.30,531.31,531.40$, 531.41, 531.50, 531.51, 531.60, 531.61, 531.70, 531.71, 531.90, 531.91, 532.00, 532.01, 532.10, 532.11, 532.20, 532.21, 532.30, 532.31, $532.40,532.41,532.50,532.51,532.60,532.61,523.70,532.71,532.90$, 532.91, 533.00, 533.01, 533.10, 533.11, 533.20, 533.21, 533.30, 533.31, 533.40, 533.41, 533.50, 533.51, 533.60, 533.61, 533.70, 533.71, 533.90, 533.91, 534.00, 534.01, 534.10, 534.11, 534.20, 534.21, 534.30, 534.31, 534.40, 534.41, 534.60, 534.61, 534.70, 534.71, 534.90, 534.91, 535.00, 535.01, 535, 535.0, 535.10, 535.11, 535.20, 535.21, 535.30, 535.31, $535.40,535.41,535.50,535.51,535.60,535.61,535.70,535.71$

$553.20,553.2,553.21,553.29,552.2,552.20,552.21,552.29,551$, $551.10,551.00)$

577.2

410, 410.0, 410.00, 410.01, 410.02, 410.10, 410.11, 410.12, 410.20, $410.21,410.22,410.30,410.40,410.41,410.42,410.50,410.51$, $410.52,410.60,410.61,410.62,410.70,410.71,410.72,410.80$, $410.82,410.90 .410 .91,410.92$

$435,435.0,435.1,435.2,435.3,435.8,235.9,436,437.1$

576.4

$820,820.0,820.00,820.01,820.02,820.03,820.09,820.10,820.11$, $820.12,820.13,820.19,820.20,820.21,820.22,820.30,820.31$, $820.32,820.8,820.9,821.00,821.10$ 
acute care hospital to another acute care hospital or rehabilitation facility. Therefore, we excluded patients who were readmitted on the same day they were discharged, assuming these represented hospital to hospital transfers. In many cases, patients were readmitted more than one time over the 1-year time period.

A record was created for each readmission including the first seven discharge diagnosis codes and the first seven procedure codes for each hospitalization. Many patients were readmitted more than one time. On examining the reasons for readmissions, it was clear that reasons for readmission were clustered among multiple readmissions in the same patient. Over $95 \%$ of multiple readmissions in a single patient were for the same or related diagnoses. For this reason, we evaluated only the first readmission. For example, if a patient was readmitted for the first time in the early time period and again in the late time period, only the readmission in the early time period was reexamined. We also evaluated late readmission using a conditional repeated events analysis, and the conclusions did not change. We report the former.

Each readmission record was independently reviewed by two authors. After examining the diagnosis and procedure codes, each readmission was assigned a primary reason for the admission. The proportion of readmissions clearly related to postoperative complications was reported. However, these were subject to the reviewers' interpretation. Therefore, we also report the frequency of specific diagnoses based on the appearance of the ICD-9 diagnosis code anytime during admission. Each readmission record was queried to identify the incidence of several specific diagnoses and procedures present. The incidence of each diagnosis or procedure in each time period was calculated by identifying the frequency of the ICD-9 codes for each respective diagnosis or procedure and dividing this number by the number of first readmissions during the same time period. These diagnoses need only be present in the readmission diagnosis codes but did not need to be the primary reason for readmission. As such, they do not add up to $100 \%$.

The ICD-9 codes used to identify specific procedures and diagnoses are shown in Table 1. Specific procedures evaluated included upper endoscopy with or without intervention and biliary drainage via a percutaneous, endoscopic, or operative approach. Specific diagnoses evaluated included operative complications, metastatic disease, dehydration, gastric outlet obstruction/delayed gastric emptying, venous thromboembolism (VTE) and/or pulmonary embolus (PE), pneumonia, cholangitis, small bowel obstruction, gastritis/duodenitis/gastric ulcer/duodenal ulcer/marginal ulcer, incisional hernia, pancreatic pseuodcyst, acute myocardial infarction, cerebrovascular accident, bile leak, and hip fracture.
Statistical Analysis

SAS version 9.1.3 (Cary, NC) was used for all statistical analyses. Descriptive statistics were reported for the patient demographics, operative details, the in-hospital and 30-day mortality, the number of patients requiring readmission (total, within 30 days, and between 30 days and 1 year), number of total readmissions in each time period, the primary reason for each readmission, and the incidence of specific diagnoses during readmissions. The diagnoses during readmission were compared between the early and late readmission groups using univariate statistics (chisquare, Fisher's exact test).

Kaplan-Meier survival curves were used to determine readmission rates over the first year. Deaths related to pancreatic cancer were censored at their time of death if death occurred within the first year of discharge and prior to any readmission. Within 30 days of discharge, there were only 13 deaths (11 occurring without a previous readmission), so death was not a significant competing event. Beyond 30 days, the number of deaths increased with time, and death became a significant competing cause. We used a Cox proportional hazards model with deaths treated as censored values to assess patient-level predictors of readmission within 30 days. Between 30 days and 1 year, we excluded patients who died or were readmitted within the first 30 days. We then used two separate Cox models: the first treated deaths within the first year as censored and the second treated deaths as a competing cause. The patient-level factors determining late readmission in each model were analyzed. The assumption of proportionality was tested using Schoenfeld residuals. Significance was accepted at the $P<0.05$ level.

\section{Results}

\section{Overall Cohort}

Between 1992 and 2003, 1,730 subjects met the inclusion criteria for the study. The demographic data, type of procedure, number of in-hospital deaths, postoperative length of stay, 30-day mortality rate (including in-hospital deaths), and stage of disease for the overall cohort are shown in Table 2. The mean age of the study population was $72.6 \pm 6.4$ years. Forty-eight percent of patients were male and $82 \%$ were white. The location of the tumor dictated the type of procedure performed. Pancreaticoduodenal resections were performed in $76 \%$ of patients, distal pancreatectomy in $18 \%$ of patients, total pancreatectomy in $3 \%$ of patients, and $3 \%$ underwent pancreatectomy not otherwise specified. The median postoperative length of stay for all patients was 14 days (25th percentile $=10$ days, 75 th percentile $=21$ days). 
Table 2 Demographics of Overall Cohort $(N=1,730)$

\begin{tabular}{|c|c|c|}
\hline & Number of patients & Percent \\
\hline Age at surgery & 1,730 & $72.6 \pm 6.4$ years \\
\hline \multicolumn{3}{|l|}{ Gender } \\
\hline Male & 822 & $48 \%$ \\
\hline Female & 908 & $52 \%$ \\
\hline \multicolumn{3}{|l|}{ Race } \\
\hline White & 1,427 & $82 \%$ \\
\hline Black & 132 & $8 \%$ \\
\hline Hispanic & 74 & $4 \%$ \\
\hline Other & 97 & $6 \%$ \\
\hline \multicolumn{3}{|l|}{ Marital status } \\
\hline Married & 1,044 & $60 \%$ \\
\hline Single & 250 & $14 \%$ \\
\hline Widowed & 380 & $22 \%$ \\
\hline Unknown & 56 & $3 \%$ \\
\hline \multicolumn{3}{|l|}{ Charlson comorbidity score } \\
\hline 0 & 1,108 & $64 \%$ \\
\hline 1 & 411 & $24 \%$ \\
\hline 2 & 138 & $8 \%$ \\
\hline 3 or more & 73 & $4 \%$ \\
\hline \multicolumn{3}{|l|}{ Type of procedure } \\
\hline Pancreaticoduodenal resection & 1,309 & $76 \%$ \\
\hline Distal pancreatectomy & 311 & $18 \%$ \\
\hline Total pancreatectomy & 62 & $3 \%$ \\
\hline Pancreatectomy, not otherwise specified & 48 & $3 \%$ \\
\hline \multicolumn{3}{|l|}{ Stage of disease } \\
\hline Locoregional & 1,493 & $86 \%$ \\
\hline Distant/unknown & 237 & $14 \%$ \\
\hline Postoperative length of stay & 1,730 & $17.5 \pm 11.6$ days \\
\hline In-hospital mortality & 130 & $7.5 \%$ \\
\hline 30-day mortality & 143 & $8.3 \%$ \\
\hline
\end{tabular}

Among the 1,730 patients, there were 130 in-hospital deaths (7.5\%) following surgery, leaving a total of 1,600 patients with the potential for readmission. The 30-day mortality was $8.3 \%$ (including in-hospital mortalities); 13 patients died after the first discharge but within 30 days.

\section{Overall Readmissions}

Of the 1,600 patients with the potential for readmission, 784 patients were readmitted a total of 1,766 times within the first year of discharge. Three hundred twenty-six patients were readmitted once, 211 patients were readmitted twice, 117 patients were readmitted three times, and 130 patients were readmitted four or more times. Figures $1 \mathrm{a}$ and $1 \mathrm{~b}$ are Kaplan-Meier curves showing the time to readmission over the first year following discharge. Operative deaths are censored in Fig. 1a and treated as competing events in Fig. 1b. In addition to the survival curve, Fig. 1c depicts the number at risk in each time period, the cumulative readmissions at the end of each time period, the cumulative deaths without readmission at the end of each time period, and the Kaplan-Meier estimates for readmission rates at the same time points. The KaplanMeier curve shows a $15.5 \%$ readmission rate at 30 days and a $52.6 \%$ readmission rate at 1 year.

The mean duration for readmission was $8.8 \pm 9.7$ days (median=6 days). Readmissions accounted for 15,409 additional hospital days in the 784 patients.

\section{Early Readmissions}

Within 30 days after discharge, 248 patients (16\%) who survived the initial hospitalization were readmitted a total of 320 times; 190 patients were readmitted once, 46 patients were readmitted twice, and 12 patients were readmitted three or more times. When evaluating the individual reason for each readmission (first readmission only), $80 \%$ were related to operative complications and 
a

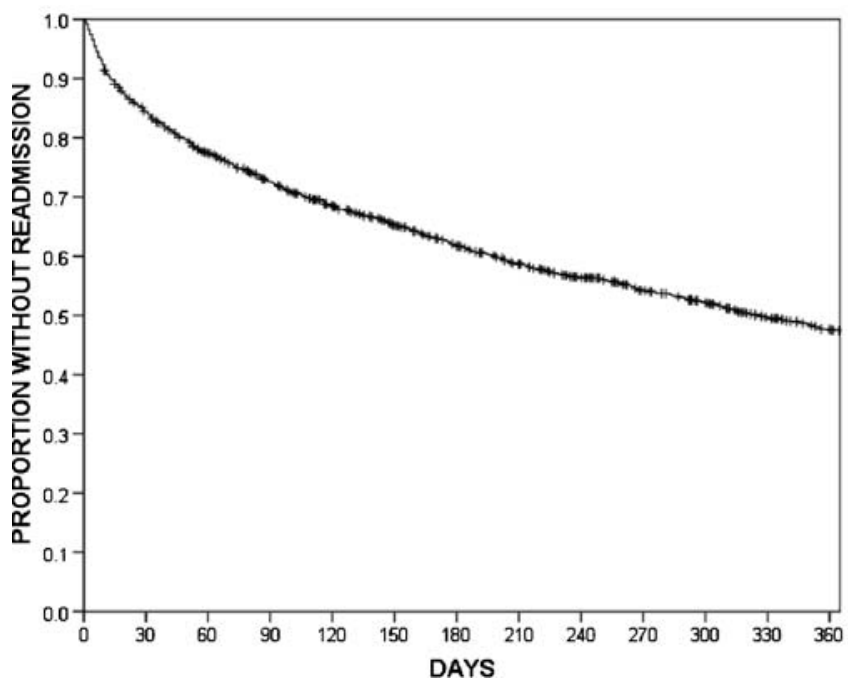

b

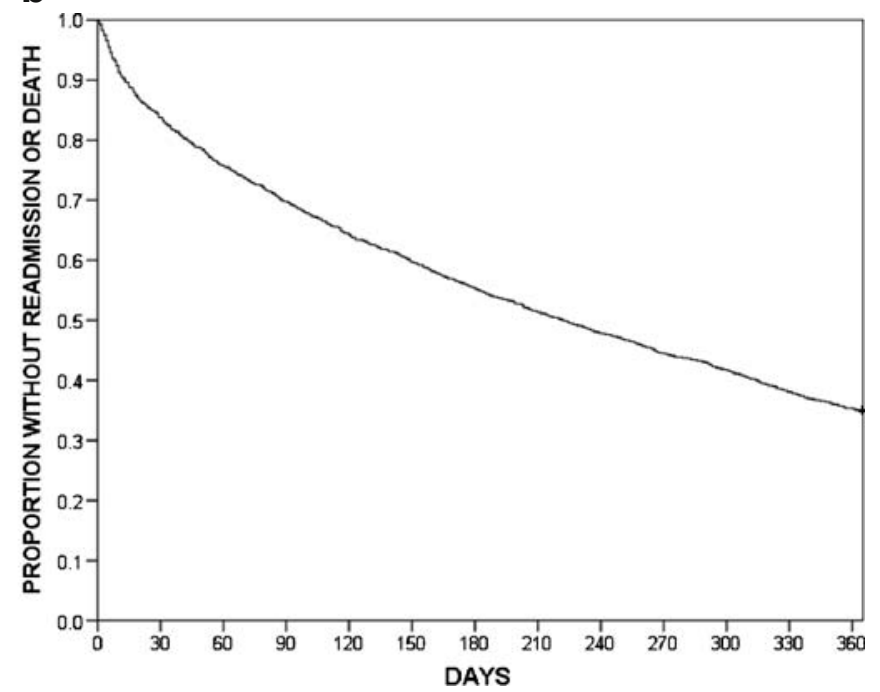

C

\begin{tabular}{|l|c|c|c|}
\hline Interval after Discharge & $\begin{array}{c}\text { Patients at Risk at } \\
\text { Beginning of Period }\end{array}$ & $\begin{array}{c}\text { Cumulative Readmissions } \\
\text { by End of Period }\end{array}$ & $\begin{array}{c}\text { Cumulative Deaths without } \\
\text { Readmission by End of Period }\end{array}$ \\
\hline & Number (Percent) & Number (Percent) & Number (Percent) \\
\hline $1-29$ days & $1600(100.0)$ & $248(15.5)$ & $29(1.7)$ \\
\hline $30-59$ days & $1341(83.8)$ & $358(22.4)$ & $50(3.1)$ \\
\hline $60-89$ days & $1213(75.8)$ & $434(27.1)$ & $130(8.1)$ \\
\hline $90-179$ days & $1116(69.8)$ & $593(37.1)$ & $266(16.6)$ \\
\hline $179-364$ days & $887(55.4)$ & $784(49.0)$ & \\
\hline$>=365$ days & $560(35.0)$ & & \\
\hline
\end{tabular}

Figure 1 Kaplan-Meier curves showing the time to readmission over the first year following initial discharge after pancreatectomy in Medicare patients. a Kaplan-Meier curve for time to readmission with deaths treated as censored. b Kaplan-Meier curve for time to readmission with deaths treated as a competing event. c The table shows the patients at risk at five different time intervals over the first year following discharge, the cumulative readmissions, and the cumulative deaths for five time periods, and the Kaplan-Meier estimates of readmission rates as well as combined readmission and death rates. included abscess, sepsis, hemorrhage, probable pancreatic fistula, GI bleed, UTI, pneumonia, respiratory failure, and VTE/PE. When looking for specific ICD-9 codes, the most common diagnosis codes noted during readmission were for operative complications (27.4\%), dehydration (27.8\%), and gastric outlet obstruction/delayed gastric emptying (8.1\%). Of the patients, $19.4 \%$ had a diagnosis code for metastatic disease, but this was often not the primary reason for admission. A complete list of diagnoses noted during early readmission is shown in Table 3 .

\section{Late Readmissions}

There were 688 patients readmitted a total of 1,446 times between 30 days and 1 year following the initial pancreatec- tomy. Of the 688 patients, 152 were also readmitted within the first 30 days following surgery, leaving 536 patients in the late group at risk for first readmission. Late readmission was related to recurrence in $48 \%$, operative complications in $25 \%$, and unrelated diagnoses in $27 \%$ of cases. The diagnoses recorded during readmission in this time period differed significantly from the reasons observed in the first 30 days following discharge (Table 3 ). The most common diagnoses during late readmissions were metastatic disease (44.0\%), dehydration (23.3\%), and VTE/PE (9.1\%).

Comparison of Reasons for Early and Late Readmissions

We compared the incidence of specific diagnoses during readmission in the early and late readmission groups. These 
Table 3 Comparison of Reasons: Early vs. Late

\begin{tabular}{|c|c|c|c|}
\hline & $\begin{array}{l}\text { Early }(N=248) \\
\text { Number }(\%)\end{array}$ & $\begin{array}{l}\text { Late }(N=536) \\
\text { Number }(\%)\end{array}$ & $\begin{array}{l}P \text { value } \\
<0.0001\end{array}$ \\
\hline Operative complications & $68(27.4)$ & $20(3.7)$ & $<0.0001$ \\
\hline Metastases & $48(19.4)$ & $236(44.0)$ & $<0.0001$ \\
\hline Dehydration & $69(27.8)$ & $125(23.3)$ & 0.17 \\
\hline Gastric outlet obstruction/delayed gastric emptying & $20(8.1)$ & $21(3.9)$ & 0.02 \\
\hline VTE/PE & $12(4.8)$ & $49(9.1)$ & 0.04 \\
\hline Pneumonia & $<11$ & $32(6.0)$ & 0.10 \\
\hline Cholangitis & $14(5.6)$ & $32(6.0)$ & 0.86 \\
\hline Small bowel obstruction & $11(4.4)$ & $35(6.5)$ & 0.24 \\
\hline Gastritis/duodenitis/gastric ulcer/duodenal ulcer/marginal ulcer & $15(6.0)$ & $34(6.3)$ & 0.87 \\
\hline Incisional hernia $^{\mathrm{a}}$ & $0(0 \%)$ & $<11(<2.1 \%)$ & 0.09 \\
\hline Pancreatic pseudocyst & $<11(<4.4 \%)$ & $<11(<2.1 \%)$ & 0.21 \\
\hline Acute myocardial infarction & $<11(<4.4 \%)$ & $<11(<2.1 \%)$ & 0.91 \\
\hline Cerebrovascular accident & $<11(<4.4 \%)$ & $<11(<2.1 \%)$ & 0.93 \\
\hline Bile leak $^{\mathrm{b}}$ & $<11(<4.4 \%)$ & $0(0 \%)$ & 0.04 \\
\hline Hip fracture ${ }^{a}$ & $0(0 \%)$ & $<11(<2.1 \%)$ & 0.03 \\
\hline Esophagogastroduodenoscopy and related procedures & $22(8.9)$ & $71(13.3)$ & 0.08 \\
\hline Biliary drainage (endoscopic, percutaneous, or operative) & $15(6.1)$ & $49(9.1)$ & 0.14 \\
\hline
\end{tabular}

${ }^{a}$ Both groups $<11$ patients but significantly higher incidence of incisional hernias and hip fractures in the late readmission group.

${ }^{\mathrm{b}}$ Both groups $<11$ patients but significantly higher incidence of bile leaks in early readmission group.

diagnoses may not have been the primary reason for readmission. When compared to patients in the late readmission group, patients readmitted early were more likely to be readmitted with a diagnosis of postoperative complications, gastric outlet obstruction/delayed gastric emptying, and pancreatic pseudocyst. They were less likely to be readmitted with metastatic disease, VTE/PE, and hip fractures (Table 3). The incidence of dehydration was similar between the two groups; however, in the late group, this diagnosis is more related to metastatic disease and failure to thrive, whereas in the early group, it is related postoperative complications.

\section{Cox Proportional Hazards Model: Factors Predicting Early Readmission}

The year of surgery, age, race, sex, marital status, income, education, Charlson comorbidity score, type of operation, complications recorded in the billing records for the initial hospitalization, postoperative length of stay $(<10$ days or $\geq 10$ days), tumor stage, and nodal status were included in all multivariate models. For early readmissions, only the type of operation and the postoperative length of stay (LOS) predicted readmission. Patients undergoing distal pancreatectomy had a hazard ratio (HR) for readmission of 1.66 (95\% CI, 1.19-2.33) when compared to those undergoing pancreaticoduodenectomy. Patients undergoing total pancreatectomy and pancreatectomy not otherwise specified had the same likelihood of readmission as patients undergoing pancreaticoduodenal resection. Patients with an initial postoperative LOS of $\geq 10$ days had a HR for readmission of 1.46 (95\% CI, 1.04-2.05). A recorded diagnosis code for operative complications during initial hospitalization (see methods) did not predict survival. The final model is shown in Table 4.

For late readmissions, deaths due to pancreatic cancer became a significant competing cause. Figure $1 b$ shows a Kaplan-Meier curve where death and readmission are both treated as events. The rate of readmission or death within the first year was $35 \%$ (Fig. 1c).

We ran multivariate Cox proportional hazards models, the first with deaths as censored values at the time of death and the second with deaths as a competing event (Table 5). Considering deaths as censored creates informative censoring, since the same factors that influence cancer deaths likely influence late readmissions. As a result, in the first model, treating deaths as censored, only Hispanic race and a Charlson score of 3 or more (Table 4) predicted readmission. In the model with death as a competing event, the presence of distant disease at the time of surgery, positive nodal status, a Charlson score of 3 or more, and an initial length of stay $\geq 10$ days predicted readmission (Table 5).

\section{Survival Analysis}

Patients readmitted within 30 days of discharge had worse median survival (median=11.8 months; 5-year survival, 
Table 4 Cox Proportional Hazards Model: Factors Associated with Early Readmission

\begin{tabular}{|c|c|c|c|}
\hline Factor (reference group) & HR & $95 \% \mathrm{CI}$ & Type $3, P$ value \\
\hline Length of stay $\geq 10$ days ( $<10$ days $)$ & 1.46 & $1.04-2.05$ & 0.03 \\
\hline Operation (pancreaticoduodenectomy) & & & 0.02 \\
\hline Distal pancreatectomy & 1.66 & $1.19-2.33$ & \\
\hline Total pancreatectomy & 1.29 & $0.67-2.46$ & \\
\hline Other pancreatectomy & 0.76 & $0.31-1.88$ & \\
\hline Operative complications initial stay (yes) & 0.98 & $0.71-1.34$ & 0.91 \\
\hline Age (per year of age) & 1.00 & $0.98-1.02$ & 0.78 \\
\hline Year of surgery (per year) & 1.04 & $1.00-1.08$ & 0.07 \\
\hline Gender (male) & 0.87 & $0.66-1.14$ & 0.30 \\
\hline Race (non-Hispanic white) & & & 0.20 \\
\hline Non-Hispanic black & 1.03 & $0.62-1.70$ & \\
\hline Hispanic & 0.29 & $0.09-0.91$ & \\
\hline Marital status (married) & & & 0.92 \\
\hline Single & 1.05 & $0.71-1.56$ & \\
\hline Widowed & 1.08 & $0.77-1.51$ & \\
\hline Highest income quartile ( $1=$ lowest $)$ & & & 0.18 \\
\hline 2 & 0.86 & $0.58-1.26$ & \\
\hline 3 & 0.67 & $0.47-1.04$ & \\
\hline 4 & 0.61 & $0.38-0.99$ & \\
\hline Highest education quartile ( $1=$ lowest $)$ & & & 0.25 \\
\hline 2 & 1.28 & $0.85-1.93$ & \\
\hline 3 & 1.31 & $0.84-2.03$ & \\
\hline 4 & 1.67 & $1.01-2.75$ & \\
\hline Charlson comorbidity score (0) & & & 0.39 \\
\hline 1 & 1.06 & $0.77-1.44$ & \\
\hline 2 & 1.45 & $0.95-2.23$ & \\
\hline 3 or more & 1.01 & $0.58-1.93$ & \\
\hline Tumor stage (distant) & 0.82 & $0.56-1.18$ & 0.28 \\
\hline Nodal status (negative) & 0.98 & $0.74-1.30$ & 0.98 \\
\hline
\end{tabular}

$18 \%$ ) than patients not requiring readmission (median= 16.5 months; 5-year survival, $18 \%, p=0.04$, Fig. 2, $N=$ 248). From the curves, you can see that this difference in mortality is early and likely attributable to postoperative complications. Patients surviving the insult of the postoperative complications have similar 5-year survival rates to those who did not suffer complications.

Readmission between 30 days and a year was associated with worse median survival (12.1 vs. 19.4 months) and 5year survival $(12 \%$ vs. $21 \%, p<0.0001$, Fig. $3, N=536$ ) when compared to those not requiring late readmission. As a quarter of late readmissions are related primarily to progression of disease requiring readmission, this is expected.

Multiple readmissions in the early time period were not correlated with survival. Multiple readmissions in the late time period were correlated with worse survival, presumably from recurrent disease requiring rehospitalization, whereas those who remained disease-free did not require hospital admission.

\section{Discussion}

Our study demonstrates an overall readmission rate of 53\% and an early (within 30 days) readmission rate of $16 \%$ after pancreatectomy for pancreatic cancer in Medicare patients. The overall readmission rate, calculated using at time-to-event analysis, is similar to the $59 \%$ readmission rate in a previous population-based study ${ }^{22}$ and higher than the reported rates in previous single-institution studies. ${ }^{16,21}$ The higher rates in population-based studies more likely represent true readmission rates in the general population. Moreover, Yermilov and colleagues $^{22}$ found that $47 \%$ of readmissions were not to the hospital performing the primary surgery. It is likely that the single-institution studies did not capture readmissions to outside hospitals and may grossly underestimate readmission rates even in their own patients. In addition, the non-timedependent methods used in previous studies will inflate the denominator or number at risk in a given period, decreasing the observed readmission rates. 
Table 5 Cox Proportional Hazards Model: Factors Associated with Early Readmission
Factor (reference group)

Model with deaths censored

Model with deaths as competing cause

HR $(95 \% \mathrm{CI})$

\begin{tabular}{|c|c|c|}
\hline & HR $(95 \% \mathrm{CI})$ & as competing cause \\
\hline \multicolumn{3}{|l|}{ Race (non-Hispanic white) } \\
\hline Non-Hispanic black & $1.21(0.88-1.67)$ & $1.13(0.85-1.50)$ \\
\hline Hispanic & $0.42(0.23-0.75)$ & $0.69(0.46-1.01)$ \\
\hline \multicolumn{3}{|l|}{ Charlson comorbidity score $(0)$} \\
\hline 1 & $1.12(0.91-1.35)$ & $1.09(0.91-1.29)$ \\
\hline 2 & $1.06(0.74-1.50)$ & $0.98(0.73-1.32)$ \\
\hline 3 or more & $1.78(1.18-2.68)$ & $1.48(1.04-2.12)$ \\
\hline Tumor stage (distant) & $0.82(0.62-1.08)$ & $0.71(0.57-0.88)$ \\
\hline Nodal status (negative) & $1.19(0.98-1.45)$ & $1.30(1.11-1.53)$ \\
\hline Length of stay $\geq 10$ days $(<10$ days $)$ & $1.14(0.91-1.44)$ & $1.23(1.02-1.49)$ \\
\hline \multicolumn{3}{|l|}{ Operation (pancreaticoduodenectomy) } \\
\hline Distal pancreatectomy & $1.07(0.83-1.38)$ & $1.16(0.95-1.43)$ \\
\hline Total pancreatectomy & $1.11(0.68-1.81)$ & $1.12(0.75-1.66)$ \\
\hline Other pancreatectomy & $1.34(0.83-2.17)$ & $1.46(0.98-2.18)$ \\
\hline Operative complications initial stay (yes) & $1.09(0.87-1.37)$ & $1.07(0.88-1.28)$ \\
\hline Age (per year of age) & $0.98(0.97-1.00)$ & $1.00(0.99-1.01)$ \\
\hline Year of surgery (per year) & $0.99(0.97-1.02)$ & $1.01(0.98-1.03)$ \\
\hline Gender (male) & $0.84(0.70-1.01)$ & $0.99(0.80-1.09)$ \\
\hline \multicolumn{3}{|l|}{ Marital status (married) } \\
\hline Single & $0.98(0.75-1.28)$ & $0.93(0.74-1.16)$ \\
\hline Widowed & $1.08(0.55-1.36)$ & $1.03(0.85-1.25)$ \\
\hline \multicolumn{3}{|l|}{ Highest income quartile ( $1=$ lowest $)$} \\
\hline 2 & $0.83(0.64-1.08)$ & $1.01(0.80-1.26)$ \\
\hline 3 & $0.80(0.60-1.07)$ & $1.01(0.79-1.29)$ \\
\hline 4 & $0.69(0.50-0.95)$ & $0.91(0.70-1.20)$ \\
\hline \multicolumn{3}{|l|}{ Highest education quartile $(1=$ lowest $)$} \\
\hline 2 & $1.27(0.96-1.66)$ & $1.10(0.87-1.38)$ \\
\hline 3 & $1.19(0.89-1.60)$ & $1.02(0.80-1.29)$ \\
\hline 4 & $1.19(0.84-1.67)$ & $1.00(0.76-1.33)$ \\
\hline
\end{tabular}

Deaths or readmissions within 30 days were excluded. The first model treats deaths within the first year as censored. The second treats death as a competing event
As hypothesized, the length of the time period elapsed since undergoing pancreatectomy determined the reason for readmission. Early readmissions were more commonly associated with postoperative complications, while late readmission after 30 days was more likely to be due to disease progression (metastases or recurrence).

Dehydration occurred in approximately one quarter of patients in both the early and late groups. When evaluating diagnosis codes concurrent with dehydration, dehydration was more commonly related to surgical complications in the early readmission group while dehydration in the late group was more commonly related to chemotherapy or recurrence of pancreatic cancer and general failure to thrive. Likewise, the nature of postoperative complications differed between the early and late groups. Postoperative complications requiring early readmission most commonly included sepsis, abscess, anastomotic leak, and acute hemorrhage, whereas late complications included small bowel obstruction, incisional hernias, biliary strictures, and cholangitis. A diagnosis of delayed gastric emptying or gastric outlet obstruction was seen in $8 \%$ of early readmissions and $4 \%$ of late readmissions. Similar to dehydration, the reasons for the delayed gastric emptying or gastric outlet obstruction differed between the early and late groups. Early delayed gastric emptying following pancreaticoduodenectomy has been reported in $10-20 \%$ of patients immediately following pancreatic resection ${ }^{3,25}$ and accounts for the majority of delayed gastric emptying or gastric outlet obstruction in the early group. In the late group, however, this diagnosis was associated with gastric outlet obstruction secondary to tumor recurrence.

Only the initial length of stay and the type of resection predicted early readmission. Those who had an initial length of stay of ten or more days were more likely to require early 


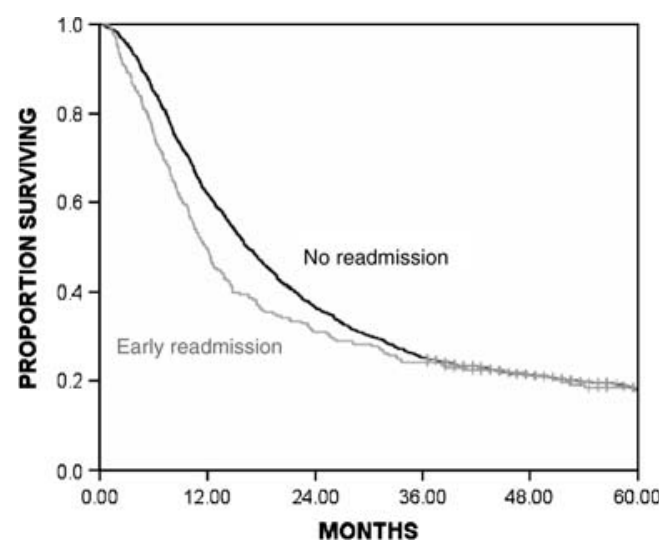

Figure 2 Kaplan-Meier actuarial survival curves showing comparing survival in patients who did not require readmission and those who were readmitted early (within 30 days, $N=248$ ). When compared to patients not readmitted, patients readmitted early had worse median survival $(11.8$ vs.16.5 months, $P=0.04)$, but the long-term survival was identical (18\%).

readmission. A diagnosis code for operative complications did not predict readmission. This suggests two things: first, not all operative complications are noted during initial admission and second, not all complications lead to readmission. A prolonged initial length of stay does not cause readmission; rather, it is likely a marker of serious postoperative complications, the most common diagnosis during early readmission. In addition, longer lengths of stay predispose patients to developing additional iatrogenic infections, as well as VTE/PE and atelectasis associated with prolonged immobility, which typically occur in a hospital setting. There was no association of age or patient comorbidities with early readmission.

This is the first study to demonstrate that patients undergoing distal pancreatectomy have an increased risk of readmission. This is unexpected as pancreaticoduodenectomy is a more complex procedure and thought to be fraught with more complications. However, pancreatic fistula rates have been reported to be higher following distal pancreatectomy than pancreatic head resection. ${ }^{26-28}$ This fact, coupled with the fact that distal pancreatectomy is less likely to be performed at high-volume centers by experienced surgeons, ${ }^{8}$ likely contribute to this finding.

In the first year after initial discharge, deaths due to pancreatic cancer became a significant competing event. Considering deaths as censored creates informative censoring, since the same factors that influence cancer deaths, likely influence late readmissions. As a result, the multivariate model evaluating the factors associated with late readmission, which treated deaths as censored, does not demonstrate the same predictive factors as the model that treats death as a competing event. By treating deaths a censored, patients with advanced tumor stage (distant disease and positive nodes) are removed from the at risk cohort. However, these factors are related to recurrence, the most common reason for readmission, and would likely have led to readmission in the absence of death.

While the median survival was lower in patients requiring early readmission compared to those who did not, the long-term survival was identical at $18 \%$, suggesting that operative complications increase early deaths. However, survivors of these complications can expect similar survival to their counterparts who had an uncomplicated postoperative course. Late readmission is more commonly due to recurrence and is a marker of early mortality. As expected, it was associated with significantly worse median and long-term survival as shown previously. ${ }^{22}$

This study has several limitations, mostly related to the use of administrative data. The reported reasons for readmission in Table 3 were based on identification of specific ICD-9 diagnosis codes both in the primary discharge diagnosis and additional diagnoses provided for the same discharge. We also individually reviewed each readmission record and looked at the diagnosis and procedure codes and gave each readmission a primary reason for the admission. The results were similar using the two methods, in that early readmissions were related to surgical complications and late readmissions were related to recurrence; however, these were subject to the reviewers' interpretation.

It is often difficult to identify specific complications commonly reported after pancreatic surgery using administrative data, including pancreatic fistula and bile leak. For example, there are codes for postoperative complications and anastomotic leak, but they are not specific. In addition, the administrative data is used for billing purposes, so diagnosis codes mandating reimbursement may be more likely to be coded. While we were able to look at nodal status, data were not available on margin status to evaluate its effect on early and late readmission.

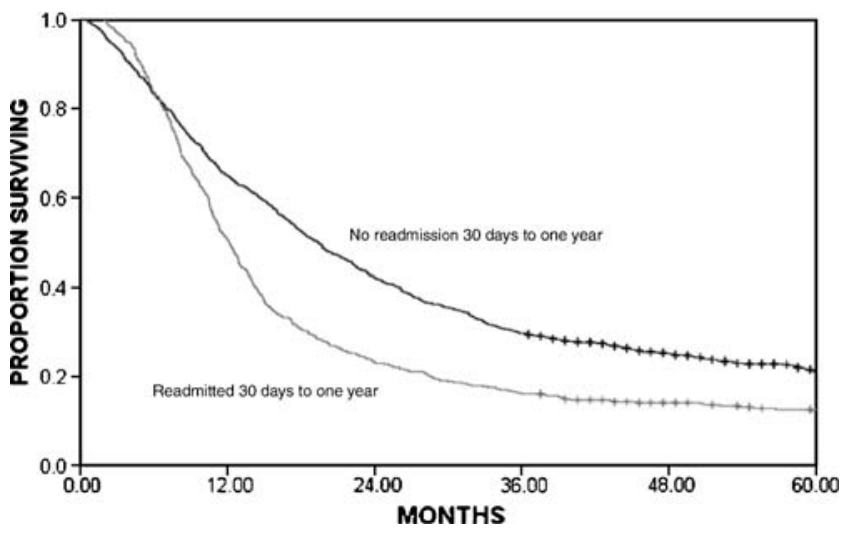

Figure 3 Kaplan-Meier actuarial survival curves showing comparing survival in patients who did not require readmission and those who were readmitted late (between 30 days and 1 year, $N=536$ ). Late readmission was associated with worse median and long-term survival (19.4 vs. 12.1 months, $21 \%$ vs. $12 \%, P<0.0001$ ). 
In summary, this study demonstrates the rates and the most frequent causes of early and late readmissions and identifies predictors of hospitalization during these time periods after initial discharge following pancreatectomy for pancreatic cancer. These findings reinforce the finding that readmission rates in the general population following pancreatectomy occur in over $50 \%$ of patients and are underreported in single-institution studies. Additionally, this study delineates the factors contributing to early and late readmissions. It demonstrates that early readmission related to complications shortens median but does not affect long-term survival if the patient survives the operative complication. Late readmissions are a marker of early mortality. Death due to cancer is a competing event with late readmission. As such, the factors influencing late readmission are similar to those that predict early mortality. The $15 \%$ of readmissions related to operative complications are, therefore, potentially preventable. The reasons for early readmissions need to be studied further to identify individual factors and operative techniques that decrease these preventable readmissions.

Acknowledgments The study used the linked SEER-Medicare database. The interpretation and reporting of these data are the sole responsibility of the authors. The authors acknowledge the efforts of the Applied Research Program (NCI), Information Management Services (IMS, Inc.), and the Surveillance, Epidemiology, and End Results (SEER) Program tumor registries in the creation of the SEERMedicare database. This work was supported in part by the Dennis W. Jahnigen Career Development Scholars Award, NIH K07 Cancer Prevention, Control, and Population Sciences Career Development Award (grant number 1K07CA130983-01A1), and Established Investigator Award (NCI-K05 CA 134923).

Open Access This article is distributed under the terms of the Creative Commons Attribution Noncommercial License which permits any noncommercial use, distribution, and reproduction in any medium, provided the original author(s) and source are credited.

\section{References}

1. Balcom JHt, Rattner DW, Warshaw AL, Chang Y, Fernandez-del Castillo C. Ten-year experience with 733 pancreatic resections: changing indications, older patients, and decreasing length of hospitalization. Arch Surg 2001;136(4):391-398.

2. Geer RJ, Brennan MF. Prognostic indicators for survival after resection of pancreatic adenocarcinoma. Am J Surg 1993;165 (1):68-72. (discussion 72-63).

3. Winter JM, Cameron JL, Campbell KA et al. 1423 pancreaticoduodenectomies for pancreatic cancer: a single-institution experience. J Gastrointest Surg 2006;10(9):1199-1210. (discussion 1210-1191).

4. Cameron JL, Riall TS, Coleman J, Belcher KA. One thousand consecutive pancreaticoduodenectomies. Ann Surg 2006;244(1):10-15.

5. Brooks AD, Marcus SG, Gradek C et al. Decreasing length of stay after pancreatoduodenectomy. Arch Surg 2000;135(7):823-830.

6. Schmidt CM, Powell ES, Yiannoutsos CT et al. Pancreaticoduodenectomy: a 20-year experience in 516 patients. Arch Surg 2004;139(7):718-725. (discussion 725-717).
7. Ho V, Heslin MJ. Effect of hospital volume and experience on inhospital mortality for pancreaticoduodenectomy. Ann Surg 2003;237(4):509-514.

8. Riall TS, Eschbach KA, Townsend CM Jr., Nealon WH, Freeman $\mathrm{JL}$, Goodwin JS. Trends and disparities in regionalization of pancreatic resection. J Gastrointest Surg 2007;11(10):1242-1251. (discussion 1251-1242).

9. Sosa JA, Bowman HM, Gordon TA et al. Importance of hospital volume in the overall management of pancreatic cancer. Ann Surg 1998;228(3):429-438.

10. Gordon TA, Burleyson GP, Tielsch JM, Cameron JL. The effects of regionalization on cost and outcome for one general high-risk surgical procedure. Ann Surg 1995;221(1):43-49.

11. Di Carlo V, Gianotti L, Balzano G, Zerbi A, Braga M. Complications of pancreatic surgery and the role of perioperative nutrition. Dig Surg 1999;16(4):320-326.

12. Goonetilleke KS, Siriwardena AK. Systematic review of perioperative nutritional supplementation in patients undergoing pancreaticoduodenectomy. JOP 2006;7(1):5-13.

13. Marandola M, Cilli T, Alessandri F et al. Perioperative management in patients undergoing pancreatic surgery: the anesthesiologist's point of view. Transplant Proc 2008;40 (4):1195-1199.

14. Kennedy EP, Rosato EL, Sauter PK et al. Initiation of a critical pathway for pancreaticoduodenectomy at an academic institutionthe first step in multidisciplinary team building. J Am Coll Surg 2007;204(5):917-923. (discussion 923-914).

15. Porter GA, Pisters PW, Mansyur C et al. Cost and utilization impact of a clinical pathway for patients undergoing pancreaticoduodenectomy. Ann Surg Oncol 2000;7(7):484-489.

16. Emick DM, Riall TS, Cameron JL et al. Hospital readmission after pancreaticoduodenectomy. J Gastrointest Surg 2006;10 (9):1243-1252. (discussion 1252-1243).

17. DeOliveira ML, Winter JM, Schafer M et al. Assessment of complications after pancreatic surgery: a novel grading system applied to 633 patients undergoing pancreaticoduodenectomy. Ann Surg 2006;244(6):931-937. (discussion 937-939).

18. Grobmyer SR, Pieracci FM, Allen PJ, Brennan MF, Jaques DP. Defining morbidity after pancreaticoduodenectomy: use of a prospective complication grading system. J Am Coll Surg 2007;204(3):356-364.

19. Kazanjian KK, Hines OJ, Eibl G, Reber HA. Management of pancreatic fistulas after pancreaticoduodenectomy: results in 437 consecutive patients. Arch Surg 2005;140(9):849-854. (discussion 854-846).

20. Satoi S, Takai S, Matsui $Y$ et al. Less morbidity after pancreaticoduodenectomy of patients with pancreatic cancer. Pancreas 2006;33(1):45-52.

21. van Geenen RC, van Gulik TM, Busch OR, de Wit LT, Obertop $\mathrm{H}$, Gouma DJ. Readmissions after pancreatoduodenectomy. Br J Surg 2001;88(11):1467-1471.

22. Yermilov I, Bentrem D, Sekeris E et al. Readmissions following pancreaticoduodenectomy for pancreas cancer: a population-based appraisal. Ann Surg Oncol 2009;16(3):554-561.

23. Surveillance, Epidemiology, and End Results. http://www.seer. cancer.gov/. Accessed February 27, 2008.

24. Warren JL, Klabunde CN, Schrag D, Bach PB, Riley GF. Overview of the SEER-Medicare data: content, research applications, and generalizability to the United States elderly population. Med Care 2002;40(8 Suppl):IV-3-IV-18.

25. Yeo CJ, Barry MK, Sauter PK et al. Erythromycin accelerates gastric emptying after pancreaticoduodenectomy. A prospective, randomized, placebo-controlled trial. Ann Surg 1993;218(3):229237. (discussion 237-228).

26. Okabayashi T, Kobayashi M, Sugimoto $\mathrm{T}$ et al. Postoperative pancreatic fistula following distal pancreatectomy for pancreatic 
neoplasm; can pancreatic fistula be prevented? Hepatogastroenterology 2004;51(60):1838-1841.

27. Goh BK, Tan YM, Chung YF et al. Critical appraisal of 232 consecutive distal pancreatectomies with emphasis on risk factors, outcome, and management of the postoperative pancreatic fistula: a 21-year experience at a single institution. Arch Surg 2008;143 (10):956-965.

28. Lillemoe KD, Kaushal S, Cameron JL, Sohn TA, Pitt HA, Yeo CJ. Distal pancreatectomy: indications and outcomes in 235 patients. Ann Surg 1999;229(5):693-698. (discussion 698-700).

Deepthi Martha Reddy, Presenter (University of Texas, Galveston, TX medical student)

\section{Discussant}

Dr. Sharon Weber (Madison): First, I have to congratulate you as a medical student in presenting this work. This is a very timely paper and I am really happy to see it presented here at the SSAT. As many of you know, CMS plans to use readmission as a quality of care indicator in the future because the estimated cost of readmissions has been estimated at about $\$ 17$ billion. We know we have underestimated the rate of readmission when utilizing single-institution studies because of readmission at other hospitals. Using the SEER-Medicare database is a great way to obtain the actual rate of readmission, so I congratulate you on this work.

I have questions surrounding two main points. First, "how can we impact this?" and the second question surrounding this issue - "Are these findings real?"

To address the first question, clearly, the mortality of almost $8 \%$ in-house and $23 \%$ at 30 days is not acceptable. In addition, the readmission rate of $16 \%$ at 30 days is also very high, considering that the median length of stay was 14 days.

Your group has presented some of the seminal work looking at hospital volume, and I am wondering if you did not look at that here. Is hospital volume one area where we may be able to impact the rate of readmission and mortality? Were high-volume hospitals less likely to have higher numbers of readmissions? In addition, was there any difference in geographic patterns for readmissions?

Secondly, a recent publication by Coleman, in the New England Journal in April 2009, examined readmissions for Medicare patients using claims data. Of those 800,000 patients who underwent both small and larger surgical procedures, the readmission rates at 30 days and 1 year were almost identical to yours. Thus, this leads to the question, "do your findings represent a real phenomenonthat the readmission is higher after pancreatectomy, which is clearly a more complex operation than the average surgical procedure?" Or do these findings just imply that the Medicare population has a higher rate of readmission overall, perhaps because of increased age?

\section{Closing discussant}

Deepthi Martha Reddy: Thank you, Dr. Weber. We did not include data on hospital volume. We did not do so because some of the hospitals at which patients might go to undergo pancreatic resection may not be included in the SEER regions. As a result, they may falsely appear as low-volume hospitals.

When we evaluated hospital volume excluding hospitals not in SEER regions, hospital volume predicted mortality, but not readmission.

For the second question, I would like to refer to my mentor Dr. Riall.

\section{Closing discussant}

Dr. Taylor S. Riall (Galveston, TX): We did not include hospital volume because some of the hospitals at which patients might undergo pancreatic resection may not be included in the SEER regions. As a result, they may falsely appear as low-volume hospitals. For instance, Johns Hopkins is a high volume hospital, but it is not in a SEER region.

If you take patients who live in New Jersey, which is a SEER region, they may travel to Baltimore to have their surgery done at Johns Hopkins. In the database, we would be able to identify Johns Hopkins as an individual hospital, but it would not appear as a high volume hospital, since we would be calculating volume based only on the number of patients living in SEER regions who had their surgery done there.

In addition, you are looking at Medicare volume and not total pancreatic resection volume. Therefore, there are inherent problems with looking at hospital volume. When we evaluated hospital volume including only hospitals in the SEER regions, hospital volume was a predictor of mortality and increased length of stay, but not readmission.

With regard to your question regarding Medicare readmission rates compared to readmission rates for pancreatectomy specifically, I think you make a good observation. Even in the single institution studies, the readmission rates are high. Therefore, I think this is actually real and not simply the readmission rates for the Medicare population. Readmission is common following pancreatectomy, and we need to evaluate the reasons for readmission and areas for improvement in a multi-institutional setting. This can be increasingly important in this pay-forperformance era.

\section{Discussant}

Dr. Keith D. Lillemoe (Indianapolis): Again, I would just echo that the medical students here put us all to shame. Great presentation. 
There is a bit of a disconnection. The 8.3\%, 30-day mortality is high. Obviously it is not a high-volume center, tertiary center, or teaching hospital. It is a national database. Regardless, it is still too high.

However, the $15 \%$ readmission rate is very acceptable. Why is there such a disconnection? Is it the fact that these people are dying before they get readmitted? I do not quite understand your data because $15 \%$ readmission is about as good as you are going to see from any of the best of institutions, whereas an $8.3 \%$ mortality is unacceptably high.

Could you explain that disconnection to me? Is it something related to the data analysis or the database that you are using?

\section{Closing discussant}

Deepthi Martha Reddy: The early the 15\% readmission rate was related to postoperative complications. This rate is likely lower than reported readmission rates since most studies look at 1 year readmission rates and not 30-day readmission rates.

\section{Discussant}

Dr. Keith Lillemoe (Indianapolis): However, do you not anticipate that the $8.3 \%$ operative mortalities are dying of postoperative complications? Those are not tumor progression for Whipples or pancreatic resections in 30 days.

\section{Discussant}

Dr. Charles Vollmer (Boston): I would like to shift gears and take it from the administrative level back down to the practice level. And the one thing that really struck me was the fact that if you are in the hospital for greater than 10 days, you have a very high chance of being readmitted soon thereafter.

These are cases where there is a deviation in the standard progression of the postoperative recovery period. And I wonder if we as surgeons can find a way to impact on that readmission rate by figuring out what we are doing wrong, or what is going on with the patient, in that first 10-day period or first stay.

Therefore, in other words, what could be predictive factors from the in-house recovery period that would say this person should not be sent home at this point? Maybe we are doing a disservice in trying to cut the length of stay days down, on some of these patients when we could tidy them up and solve the problems by keeping them in the hospital longer. Any thoughts?

\section{Closing discussant}

Dr. Taylor S. Riall: I personally think we are seeing these readmissions when we do not recognize postoperative complications. When you look at the readmission rates before and after initiation of critical pathways, you see decreasing length of stay and the readmission rates actually go down.

Therefore, I do not think the answer is to keep those people there longer to prevent the complications but, as you suggest, to identify the ones who have occult problems and need to stay. I think the patients that get readmitted are the occult complications that we do not recognize. For example, we might miss a pancreatic fistula that did not show up in the drains, so the patient appears to be "on the pathway." Then, we send them home, and they develop an abscess. I am not sure we are going to be able to reduce our readmission rates to zero, but I think it would be beneficial for high-volume centers to pool our data and identify factors predictive of readmission. This could potentially cut down readmissions and cost significantly.

I think one way to do it is to continue to centralize pancreatic resection at high volume centers. We could incorporate these predictive factors into our pathways.

\section{Discussant}

Dr. Henry Pitt (Indianapolis, IN): We have the NSQIP data from 2005-2007 on 2,000 pancreatectomies, and the mortality is less than $3 \%$ in that data base.

\section{Closing discussant}

Dr. Taylor S. Riall: This is Medicare data, and I suspect the higher observed operative mortality is expected. Increased mortality following pancreatic surgery in elderly patients has been well documented, so I would expect a higher rate in this data set than NSQIP, which includes patients of all ages and resections done for benign disease. 